\section{Vertical Concentration Profiles in Colloidal Fluids Measured by FTIR-ATR Spectroscopy}

\author{
Ben H. Erné, ${ }^{*}, \dagger$ J oop H. van der Maas, ${ }^{\ddagger}$ \\ Bonny W. M. Kuipers, ${ }^{\dagger}$ Tom Visser, ${ }^{\ddagger}$ and Albert P. Philipse ${ }^{\dagger}$ \\ Van't Hoff Laboratory for Physical and Colloid Chemistry, \\ and Department of Inorganic Chemistry and Catalysis, \\ Debye Institute, Utrecht University, Padualaan 8, \\ $3584 \mathrm{CH}$ Utrecht, The Netherlands
}

Received October 8, 2002. In Final Form: J anuary 9, 2003

\section{Introduction}

Phaseseparation or sedimentation under gravity occurs in a wide variety of colloidal fluids. ${ }^{1}$ An essential feature of these phenomena is the height dependence of the concentrations of the colloidal components. However, the measurement of such concentration profiles is not an easy task. In the study of sedimentation, one approach is to perform height-resolved light scattering measurements. From such measurements on nearly monodisperse latex spheres, Piazza et al. ${ }^{2}$ could derive the hard-sphere equation of state. Mengual et al. ${ }^{3}$ introduced a new technique (the "TURBISCAN") which can even be used with highly turbid systems, measuring both the transmitted and backscattered light intensities. However, two limitations of the light scattering approach are that the interpretation is model-dependent and that it seems to be limited to nonabsorbing systems. In the study of phase separation, the concentrations of different components in two coexisting phases have, to our knowl edge, not been determined in situ. Colloidal phase diagrams have been determined by dispersing different amounts of colloids and visually inspecting the number of phases, ${ }^{4}$ measuring their vol umes, ${ }^{5}$ or analyzing their concentrations ex situ. ${ }^{6}$ Here, weintroduceheight-resolved FTIR-ATR, a technique with which the concentrations of different colloidal components can also be measured in highly absorbing systems such as ferrofluids. The Fourier transform infrared (FTIR) spectrum of a colloidal dispersion is recorded as a function of height, using the attenuated total-internal-reflection geometry (ATR).

Infrared spectroscopy is a long-standing technique for the analysis of liquids. ${ }^{7}$ Different chemical species can be distinguished from the energies at which their vibrating

* To whom correspondence should be addressed. E-mail: b.erne@chem.uu.nl.

† Van't Hoff Laboratory for Physical and Colloid Chemistry.

₹ Department of Inorganic Chemistry and Catalysis.

(1) (a) Russel, W. B.; Saville, D. A.; Schowalter, W. R. Colloidal Dispersions; CambridgeUniversity Press: Cambridge, 1989. (b) Philipse A. P. Curr. Opin. Colloid Interface Sci. 1997, 2, 200.

(2) Piazza, R.; Bellini, T.; Degiorgio, V. Phys. Rev. Lett. 1993, 71, 4267.

(3) Mengual, O.; Meunier, G.; Cayre, I.; Puech, K.; Snabre, P. Colloids Surf., A 1999, 152, 111.

(4) (a) Vincent, B.; Edwards, J .; Emmett, S.; Croot, R. Colloids Surf. 1988, 31, 267. (b) Pusey, P. N.; Pirie, A. D.; Poon, W. C. K. Physica A 1993, 201, 322. (c) Ilett, S. M.; Orrock, A.; Poon, W. C. K.; Pusey, P. N. Phys. Rev. E 1995, 51, 1344. (d) Renth, F.; Poon, W. C. K.; Evans, R. M. L. Phys. Rev. E 2001, 64, 031402.

(5) (a) Bodnár, I.; Oosterbaan, W. D.J . Chem. Phys. 1997, 106, 7777. (b) de Hoog, E. H. A.; Lekkerkerker, H. N. W. J . Phys. Chem. B 1999, $103,5274$.

(6) (a) Gast, A. P.; Russel, W. B.; Hall, C. K. J . Colloid Interface Sci. 1986, 109, 161. (b) Leal Calderon, F.; Bibette, J .; Bias, J . Europhys. Lett. 1993, 23, 653.

(7) Stewart, J . E. Infrared Spectroscopy: Experimental Methods and Techniques; Marcel Dekker: New York, 1970.

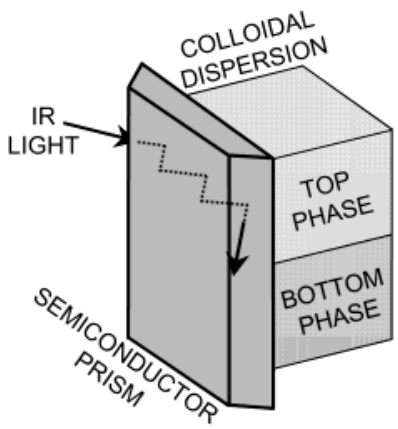

Figure 1. Schematic representation of the FTIR-ATR cell.

chemi cal bonds absorb. ATR spectroscopy is based on the interaction of light with matter at the boundary of two media with different refractive indices. In the ATR geometry, infrared light fully reflects internally insidean infrared-transparent crystal of high refractive index brought into physical contact with theliquid. Total internal reflection occurs because the angle of incidence exceeds a critical value (Mohammadi ${ }^{8}$ proposed a highly sensitive method of measuring this critical angle to determine the index of refraction of colloidal dispersions). The light intensity is not abruptly zero on the liquid side of the crystal/liquid interface, but it drops exponentially across a distance which is a fraction of the optical wavelength. Theradiation thus more or less penetrates into the liquid before it is reflected, and its intensity is attenuated due to absorption by the liquid. ${ }^{9}$ Because of this sensitivity to the near-surface region, FTIR-ATR can for instance be used to study the surface (electro)chemistry of semiconductors ${ }^{10}$ or the surface chemistry of colloids adsorbed to semi conductors, ${ }^{11}$ provided that the semi conductors can be used as an internal-reflection element. In most commercial FTIR-ATR cells for the analysis of liquids, the crystal/liquid interfaceis horizontal , but weimplemented a vertical configuration allowing measurements as a function of altitude in the gravitational field. The short, reproducible optical probe depth of the ATR method has the advantage that quantitative measurements are possible on highly absorbing samples. ${ }^{9}$ The systems studied here are opaque to the naked eye even after 100-fold dilution, the ferrofluid being ink-black and the silica dispersion milky-white.

\section{Experimental Section}

Figure 1 illustrates the principle of the method. A semiconductor ATR prism is one of the sidewalls of a cell, which holds the colloidal dispersion. Infrared light is focused at a certain height on one of the two beveled vertical sides of the prism. Light propagates horizontally inside the infrared-transparent prism, making several total internal reflections. The light is detected as it comes out of the opposite bevel ed side of the prism. U pon each reflection at thesemi conductor/liquid interface, absorbance

(8) Mohammadi, M. Adv. Colloid Interface Sci. 1995, 62, 17.

(9) (a) Harrick, N. J . Internal Reflection Spectroscopy; Wiley: New York, 1967. (b) Mirabella, F. M., J r.; Harrick, N. J . Internal Reflection Spectroscopy: Review and Supplement; Marcel Dekker: New York, 1985. (c) Hind, A. R.; Bhargava, S. K.; McKinnon, A. Adv. Colloid Interface Sci. 2001, 93, 91.

(10) (a) Chazalviel, J .-N.; Erné, B. H.; Maroun, F.; Ozanam, F.J . Electroanal. Chem. 2001, 509, 108. (b) Chabal, Y. J . Surf. Sci. Rep. 1988, 8, 211.

(11) F ourier Transform I nfrared Spectroscopy in Coll oi d and I nterface Science; Scheuing, D. R., Ed.; ACS Symposium Series 447; American Chemical Society: Washington, DC, 1991. 
occurs by chemical species near the interface. By using a FTIR spectrometer, spectra areobtained which allow thesimultaneous detection of different species at different wavenumbers. Vertical profiles are obtained by varying the height at which theinfrared light is focused.

The prism was made from a 2.0-mm-thick two-side-polished high-resistivity $\left(10^{3} \Omega \mathrm{cm}\right)$ silicon wafer grown by the float-zone technique (ITME, Poland). This material combines sufficient transmission in the infrared range with resistance against most nonoxidizing chemicals. A $15 \times 40 \mathrm{~mm}$ rectangle was cut from the wafer, and thelong sides were polished at angl es of $45^{\circ}$. The prism was used to replace one of the windows of a rectangular glass cell, using a T eflon seal, with an area of $9 \times 31 \mathrm{~mm}$ exposed tothecol loidal dispersion $(\approx 2.25$ reflections at thesemiconductor/ liquid interface). The col limated light beam of the spectrometer was focused on a 1.0-mm-high rectangular hol e in a metal plate in front of one beveled side of the prism; before being detected, the light coming out of the prism passed through a second 1.0$\mathrm{mm}$-high rectangular hole at the same height in a second metal plate. It was verified that the height resolution obtained in this way was $1.0 \mathrm{~mm}$. Thepart of theliquid probed extended a distance of about $\lambda / 25$ from the window surface $(0.4 \mu \mathrm{m}$ at a wavenumber of $\left.1000 \mathrm{~cm}^{-1}\right){ }^{12}$

TheFTIR spectrometer was a Perkin-EImer 2000 with a Globar as the infrared source and a DTGS detector operating at room temperature. Theabsorbanceat a given wavenumber and height is defined as $-\log _{10}\left[T / T_{\text {ref }}\right]$, where $T$ is the transmission of the colloidal dispersion and $\mathrm{T}_{\text {ref }}$ is the transmission of pure solvent. According to Lambert-Beer's law, ${ }^{7}$ the absorbance consists of different contributions $L \epsilon_{i} C_{i}$ of chemical components $i$, where $L$ is the optical path length, $\mathrm{C}_{\mathrm{i}}$ is the concentration of $\mathrm{i}$, and $\epsilon_{\mathrm{i}}$ is its extinction coefficient at the given wavenumber.

\section{Results and Discussion}

Theinfrared spectra A and B in Figure 2 were obtained at two different heights in a ferrofluid. The dispersion contained the solvent toluene, magnetite particles $\left(\mathrm{Fe}_{3} \mathrm{O}_{4}\right.$, $9 \pm 2 \mathrm{~nm}$ in diameter, sterically stabilized by an ol eic acid surface layer), and free poly(dimethyl siloxane) polymer (41.5 kg mol-1, radius of gyration $\approx 8.5 \mathrm{~nm}$ ). Phase separation in this system was investigated before, using a magnetic sensing coil. ${ }^{13}$ As was done in that study, samples were left to equili brate for about $16 \mathrm{~h}$ to obtain a steady-stateheight profile. Thecontributions of different chemical components to the infrared spectrum are easily distinguished (compare with reference spectra $C-E$ ). Spectrum $C$ of the polymer is dominated by peaks at 1261 $\mathrm{cm}^{-1}\left(\mathrm{Si}-\mathrm{CH}_{3}\right.$ bending mode $\left.{ }^{14}\right), 1096$ and $1016 \mathrm{~cm}^{-1}(\mathrm{Si}-\mathrm{O}$ stretching modes $\left.{ }^{14}\right)$, and $804 \mathrm{~cm}^{-1}\left(\mathrm{CH}_{3}\right.$ rocking mode $\left.{ }^{14}\right)$. The magnetite particles (spectrum $\mathrm{D}$ ) have $\mathrm{Fe}-\mathrm{O}$ vibrational modes ${ }^{15}$ bel ow $750 \mathrm{~cm}^{-1}, \mathrm{C}-\mathrm{H}$ modes of chemisorbed ol eic acid at 2926 and $2854 \mathrm{~cm}^{-1}$, and peaks at 1530 and $1440 \mathrm{~cm}^{-1}$, ascribed to stretching modes of carboxylate groups $^{16}$ obtained when oleic acid becomes attached to the magnetite surface. ${ }^{17}$ Theabsence of a strong carbonyl vibration at $1710 \mathrm{~cm}^{-1}$ (spectrumE) indicates that al most nofree ol eic acid is present in the fer rofluid. ${ }^{16}$ The bottom of the ferrofluid is clearly magnetiterich and polymerpoor, whereas the top is magnetite-poor and polymerrich. The ratio of the absorbance by oleic acid surface

(12) The intensity $E^{2}$ of the electric field $E$ drops by a factor of $e^{-1}$ at a distance equal to $(\lambda / 4 \pi)\left(n_{1}^{2} \sin ^{2} \theta-n_{2}^{2}\right)^{-1 / 2}$, where $\lambda$ is the optical wavel ength, $\theta$ is the angle of reflection $\left(45^{\circ}\right), \mathrm{n}_{1}$ is the refractive index of silicon (3.45), and $n_{2}$ is that of the liquid ( 1.50 for toluene, 1.36 for ethanol) (ref 9a).

(13) van Ewijk, G. A.; Vroege, G. J .; Kuipers, B. W. M. Langmuir 2002, 18, 382.

(14) Tsao, M.-W.; Pfeifer, K.-H.; Rabolt, J . F.; Gastner, D. G.; Häussling, L.; Ringsdorf, H. Macromolecules 1997, 30, 5913.

(15) Gasparov, L. V.; Tanner, D. B.; Romero, D. B.; Berger, H. Margaritondo, G.; Forró, L. Phys. Rev. B 2000, 62, 7939.

(16) Buckland, A. D.; Rochester, C. H.; Topham, S. A.J . Chem. Soc., Faraday Trans. 1 1980, 76, 302.

(17) Bica, D. Rom. Rep. Phys. 1995, 47, 265.

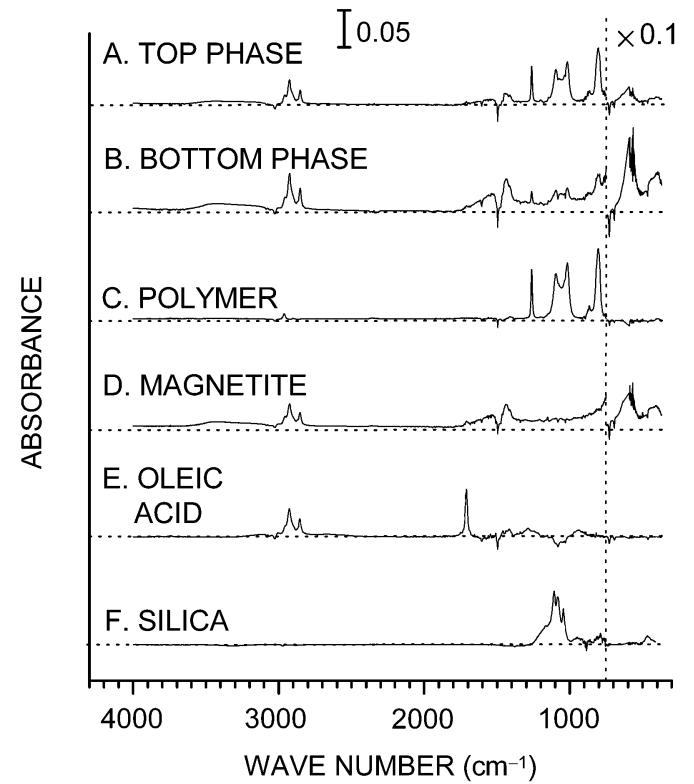

Figure 2. I nfrared spectra obtained using the vertical ATR prism. (A) and (B) were measured at different heights in a phase-separated ferrofluid. For comparison, (C) is a $130 \mathrm{~g} / \mathrm{L}$ poly(dimethyl siloxane) solution in toluene, (D) is a $890 \mathrm{~g} / \mathrm{L}$ magnetite dispersion in toluene, and (E) is a 22 vol \% solution of ol eic acid in toluene. $(F)$ is the silica dispersion used in the sedimentati on experiments. Thevertical scal ebar indicates an absorbance of 0.05 per 2.25 reflections ( 10 times less sensitive scale bel ow $750 \mathrm{~cm}^{-1}$ ).

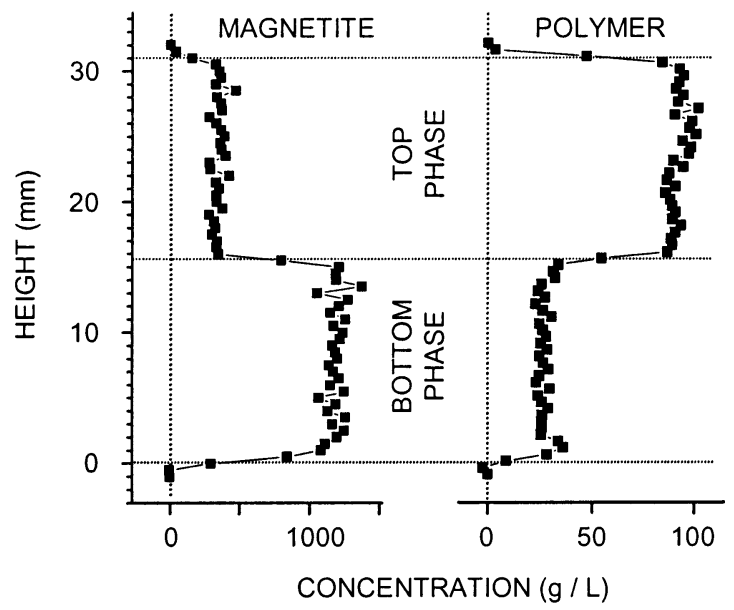

Figure 3. Vertical concentration profiles of magnetite and free polymer in a phase-separated ferrofluid.

groups to the absorbance by magnetite indicates that the particles at the top of the ferrofluid have a higher specific surface area than the (larger) particles at the bottom. Infrared spectroscopy appears to bea convenient technique to study size fractionation in this polydisperse system, an effect which severely complicates the study of phase separation using a magnetic sensing coil. ${ }^{13}$

Vertical concentration profiles of the ferrofluid are shown in Figure 3. The vertical axis gives the height in the dispersion (bottom and top at 0 and $31 \mathrm{~mm}$; the distance scanned along the prism is slightly longer, see Figure 1). The spectra obtained at each height werefitted as linear combinations of referencespectra C and D (Figure 2), obtained for homogeneous samples of known concentration (fit range, $1350-370 \mathrm{~cm}^{-1}$ ). The linearity of the absorbance of magnetite and polymer as a function of concentration was verified. This fit method is chosen here for its simplicity, to illustrate the technique; a more 


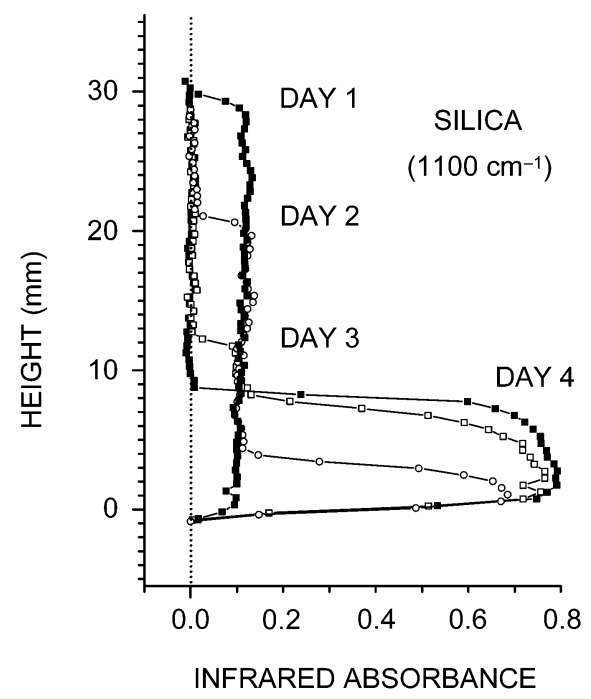

Figure 4. Vertical profiles of the absorbance at $1100 \mathrm{~cm}^{-1}$ of silica particles dispersed in ethanol before (day 1) and after sedimentation (day 4).

completeanal ysis of the data would take into account size fractionation (by separately fitting the contributions of the magnetite core and the adsorbed surface layer of the colloid particles). The scatter of the points is due to the low signal-to-noise ratio at low wavenumbers, which still has to be improved. Thecurves in Figure 3 areequilibrium profiles and show phaseseparation with a sharp interface. The phase separation is due to the depletion interaction, a well-known entropy-driven effect when a concentrated dispersion of colloi dal particles is mixed with nonadsorbing polymer molecules of similar radius of gyration. ${ }^{18}$

The height profiles in Figure 4 are of silica spheres dispersed in ethanol which takeabout 4 days to sediment to the bottom of the cell at $20^{\circ} \mathrm{C}$ (spheres with a diameter of $880 \mathrm{~nm}$, stabilized by a surface layer of 3-methacryloxypropyltrimethoxysilane, ${ }^{19}$ initial silica volumefraction $=14 \%$ ). The sedimentation rate was about 3 times as slow as theStokes velocity for noninteracting particles at infinitedilution. ${ }^{1}$ This hindered settling is duetorepulsion between the negatively charged silica particles, as discussed in detail elsewhere. ${ }^{19}$ The infrared spectrum (Figure 2F) is dominated by $\mathrm{Si}-\mathrm{O}$-related modes ${ }^{20}$ bel ow $1300 \mathrm{~cm}^{-1}$; the wavenumber-independent baseline above $1300 \mathrm{~cm}^{-1}$ indicates that optical scattering is negligible in this range. As the particles sediment, the average absorbance of the entire sample at $1100 \mathrm{~cm}^{-1}$ almost doubles (Figure 4). The main reason is that the infrared probe depth is smaller than the particles ${ }^{12}$ and that the detected silica particlesare, on average, closer totheprism wall in the sediment than in the initial dispersion. Absorbance increases less at lower wavenumbers, where the infrared probe depth is larger. ${ }^{12}$

The limitations of the method are partly related to the fact that only a thin layer of colloidal dispersion near the

(18) (a) Asakura, S.; Oosawa, F. J . Chem. Phys. 1954, 22, 1255. (b) Vrij, A. Pure Appl. Chem. 1976, 48, 471.

(19) Thies-Weesie, D. M. E.; Philipse, A. P.; Nägele, G.; Mandl, B. Klein, R. J . Colloid Interface Sci. 1995, 176, 43.

(20) Pasquarello, A.; Car, R. Phys. Rev. Lett. 1997, 79, 1766. cell wall is analyzed. For straightforward interpretation, the first micrometer of the dispersion must be representative of the bulk, and surface effects such as adsorption of the colloidal particles to the prism should be negl igible. This is the case for concentrated dispersions of nanoparticles with an organicstabilization layer such as in Figure 3, but not for particles Iarger than the penetration depth of the $\mathrm{R}$ radiation, such as the micrometer-sized particles of Figure 4. Other authors have used an evanescent wave of visiblelaser light tostudy theinteraction of a flat surface with single colloidal particles of several micrometers. ${ }^{21}$ Another difficulty is that, in principle, the penetration depth of the IR light depends on the concentrations of the chemical components, which affect the refractive index and can shorten the probe depth by absorption. ${ }^{9}$ Quantitative measurements therefore require calibration measurements on homogeneous dispersions with known concentrations. Finally, the colloidal components whose concentrations are to be determined should have sufficiently distinct infrared spectra. It is difficult to separate the spectral contributions of various organic molecules with similar chemical groups. Thetechniqueappears well suited to study the phase behavior of colloidal systems with a small number of different components, for instance, two types of inorganic nanoparticles dispersed in an organic solvent.

\section{Conclusion}

Height-resolved FTIR-ATR enables the in situ measurement of vertical concentration profiles in inhomoge neous colloidal fluids. At first approximation, infrared absor banceis proportional to concentration, sothat it can be seen as a linear way of counting particles. Different colloi dal components can beanalyzed si multaneousl y from their different contributions to the infrared spectrum. To our knowledge, Figure 3 presents the first in situ measurements of concentrations of colloi dal particles and polymer molecules in two coexisting colloidal phases as a function of height. A wide range of systems can in principle be studied, because almost all chemical species absorb in the vibrational infrared range and the infrared spectrum is different for each species, especially when they contain different chemical groups. N ot only organic molecules but also solid inorganic particles dispersed in a solvent can be investigated. An extremely high absorbance or turbidity is not a restriction, as demonstrated by the presented data. Soon, the method could for instance be used to gain new insight into the phase behavior and sizefractionation of ferrofluids. Ferrofluids are of current interest as thestarting point for preparing magnetic selfassembly structures, a wet process which puts stringent requirements on the monodispersity of the magnetic particles. $^{22}$

Acknowledgment. Wethank Dr.J .-N. Chazalviel and Dr. F. Ozanam (E cole Polytechnique, F rance) for hel pful discussions and G. Harder, P. de Graaf, and P. Engels for constructing the setup.

\section{LA026667B}

(21) Walz, J . Y. Curr. Opin. Colloid Interface Sci. 1997, 2, 600.

(22) Sun, S.; Murray, C. B.; Weller, D.; Folks, L.; Moser, A. Science 2000, 287, 1989 . 